\title{
Intervalo hídrico ótimo e produtividade de cultivares de soja
}

\author{
Amauri N. Beutler ${ }^{1}$, José F. Centurion ${ }^{1}$, Alvaro P. da Silva ${ }^{2} \&$ José C. Barbosa ${ }^{1}$
}

\begin{abstract}
RESUMO
Determinar o intervalo hídrico ótimo e sua relação com a produtividade de soja, foi o que se objetivou com o presente estudo, razão por que se conduziu um experimento em Jaboticabal, SP, em Latossolo Vermelho de textura média. O delineamento experimental foi inteiramente casualizado, em parcelas subdivididas (seis níveis de compactação e quatro cultivares), com quatro repetições. Os níveis de compactação, foram: $T_{0}=0 ; T_{1^{*}}=1 ; T_{1}=1 ; T_{2}=2 ; T_{4}=4$ e $T_{6}=6$ passadas, no mesmo local, de um trator de 11 toneladas, perfazendo toda a superfície do solo. No tratamento $T_{1 *}$ a compactação ocorreu quando o solo estava mais seco. Em dezembro de 2003 foram semeadas as cultivares de soja (Glycine max) IAC Foscarin 31, MG/BR 46 (Conquista), BRS/MG 68 (Vencedora) e IAC 8 - 2. Após a semeadura, coletaram-se amostras indeformadas de solo nas camadas de 0,03-0,06; 0,08-0,11; 0,15-0,18; e 0,22-0,25 m, para determinação da curva de retenção de água, de resistência do solo à penetração e do intervalo hídrico ótimo (IHO). Segundo o modelo do $\mathrm{IHO}$, a densidade do solo crítica (Dsc) à produtividade de soja varia entre as cultivares nos valores de 1,56 a 1,64 $\mathrm{Mg} \mathrm{m}^{-3}$. A densidade do solo, a partir da qual a produtividade das cultivares de soja decresceu, foi superior à Dsc.
\end{abstract}

Palavras-chave: atributos físicos, conteúdo de água, Glycine Max

\section{Optimal water interval and yield of soybean cultivars}

\begin{abstract}
The objective of this study was to determine the optimal water interval and its relationship with soybean yield. For this study, an experiment was carried out in Jaboticabal, São Paulo State, Brazil, in a Haplustox medium textured soil. The experimental design consisted of a completely randomized with subdivided plots (six compaction levels and four soybean cultivars), with four replications. The soil compaction levels were: $T_{0}=0, T_{1^{*}}=1, T_{1}=1, T_{2}=2$, $\mathrm{T}_{4}=4$ and $\mathrm{T}_{6}=6$ strides of a 11 ton tractor, on the same place, side by side on soil surface. $\ln \mathrm{T}_{1^{*}}$ the compaction occurred when the soil was dry. In December, 2003 the soybean (Glycine max), cultivars IAC Foscarin 31, MG/BR 46 (Conquista), BRS/MG 68 (Vencedora) and IAC 8 - 2 were sown. After sowing, indeformed soil samples were collected in layers of $0.03-0.06,0.08-0.11,0.15-0.18$ and $0.22-0.25 \mathrm{~m}$ for determination of soil water content and resistance to penetration curve and the optimal water content. According to model, the critical soil bulk density (Dsc) for soybean yield varied between cultivars from 1.56 to $1.64 \mathrm{Mg} \mathrm{m}^{-3}$. The soil bulk density at which the yield of soybean cultivars decreased was greater than the Dsc.
\end{abstract}

Key words: physical attributes, water content, Glycine max

${ }^{1}$ FCAV/UNESP, Via de Acesso Paulo Donato Castellane, s/n, CEP 14870-000 Jaboticabal, SP. Fone: (16) 3209-2672; (16) 3209-2624. E-mail: amaurib@yahoo.com.br; jfcentur@fcav.unesp.br; jcbarbosa@fcav.unesp.br

${ }^{2}$ ESALQ/USP, CP 09, CEP 13418-900, Piracicaba, SP. Fone: (19) 3429-4171. E-mail: apisilva@carpa.ciagri.usp.br 


\section{INTRODUÇÃO}

A utilização dos solos para fins agrícolas, causa alteração estrutural, com maior ou menor grau, em conseqüência da intensidade e forma de uso e manejo. Esta alteração e/ou degradação estrutural se caracteriza pela redução da porosidade total em resposta ao pequeno aumento da microporosidade e detrimento acentuado dos poros de maior diâmetro (Dexter, 2004). Este fenômeno promove aumento da densidade e da resistência do solo, refletindo-se em prejuízosàa dinâmica da água e nutrientes e ao crescimento das raízes (Letey, 1985) e, frequentemente, na redução da produtividade das culturas (Beutler et al., 2004). A alteração da estrutura do solo, pela ação antrópica leva, em geral, à limitação da qualidade física para o desenvolvimento radicular. Referida inconveniente alteração se intensifica com o tráfego contínuo de máquinas e baixa freqüência ou ausência de revolvimento do solo, como no sistema plantio direto de soja, cuja área é incrementada anualmente, no Brasil.

Desta forma, a avaliação e o monitoramento da qualidade física têm sido intensificados e realizados por meio de atributos físicos, como a densidade, porosidade, estabilidade de agregados, infiltração e retenção de água do solo (Nelson et al., 1975; Ekwue \& Stone, 1995); no entanto, é difícil estabelecer uma relação funcional desses atributos com a produtividade das culturas (Boone, 1986).

O desenvolvimento adequado das plantas, dentre outras variáveis, é função da disponibilidade de água, contato soloraízes, espaço poroso suficiente ao movimento de água, nutrientes e gases e resistência à penetração que não comprometa o crescimento radicular. Desta forma, o conteúdo de água, a aeração e a resistência do solo à penetração, prejudicam diretamente o crescimento radicular e a produtividade das culturas (Letey, 1985). A partir destas variáveis, o autor desenvolveu um modelo para integrar esses atributos físicos em um único parâmetro. Posteriormente, foi aprimorado por Silva et al. (1994) e introduzido no Brasil por Tormena et al. (1998) e denominado intervalo hídrico ótimo (IHO); trata-se de um modelo que define a amplitude do conteúdo de água em que o solo apresenta qualidade física adequada ao crescimento radicular, por não indicar limitações de água, aeração e impedimento mecânico ao crescimento radicular; define, também, a partir de que valor de densidade do solo (Ds) o IHO é zero. Quando este valor de Ds é atingido, é chamado densidade do solo crítica (Dsc) ao crescimento radicular, quando o solo apresenta condições físicas restritivas, independente do conteúdo de água.

O IHO é definido pelo limite superior e inferior de conteúdos de água no solo, considerados limitantes ao crescimento radicular. O limite superior é o menor valor entre o conteúdo de água na porosidade de aeração de 10\% (Grable \& Siemer, 1968; Zou et al., 2000) e o conteúdo de água retida na capacidade de campo, tensão de 100 hPa (Reichardt, 1988); e o limite inferior é o maior valor entre o conteúdo de água retida no ponto de murcha permanente, na tensão de 15000 hPa (Savage et al., 1996) e o conteúdo de água a partir do qual a resistência do solo à penetração (RP) é crítica ao crescimento radicular.
Sendo os valores dos atributos físicos utilizados na quantificação do IHO críticos ao crescimento radicular e se o conteúdo de água está nos limites descritos, o solo apresenta qualidade física e poucas restrições ao crescimento das plantas; assim, Silva \& Kay (1996) estudaram a relação do IHO com a produtividade de milho e verificaram que o crescimento das plantas foi positivamente correlacionado com o IHO, ou seja, a produtividade foi maior quando o conteúdo de água permaneceu dentro dos limites adequados do IHO. Em solos tropicais, no Brasil, Beutler et al. (2004), avaliaram a qualidade física do solo para a produção de soja e arroz de sequeiro, em casa de vegetação, utilizando o IHO e constataram que este não só apresentou relação positiva com a produtividade dessas culturas, mas variou com o tipo de solo. Leão et al. (2004) verificaram que o IHO mostrou condições físicas mais restritivas ao crescimento de pastagens, quando o solo foi submetido a uma lotação animal maior por área.

Esses estudos indicam que o intervalo hídrico ótimo tem relação funcional com a produtividade das culturas no campo e, para testar esta hipótese, realizou-se um estudo com o objetivo de determinar o intervalo hídrico ótimo e sua relação com a produtividade de cultivares soja.

\section{MATERIAL E MÉTODOS}

O experimento foi realizado em Jaboticabal, SP (21 15' 29” S e 48 16' 53” W; 600 m de altitude), que possui clima do tipo Cwa, segundo classificação de Köppen. Utilizou-se o Latossolo Vermelho distrófico, típico, textura média, A moderado, caulinítico, hipoférrico (LVd), cuja composição granulométrica foi determinada em amostras deformadas, por meio da dispersão com $\mathrm{NaOH}\left(0,1 \mathrm{~mol} \mathrm{~L}^{-1}\right)$ e agitação lenta durante $16 \mathrm{~h}$, sendo a argila obtida pelo método da pipeta (Gee \& Bauder, 1986). Na camada de 0,0 0,20 m, o LVd apresentou valores médios de 330, 35 e $635 \mathrm{~g} \mathrm{~kg}^{-1}$ de argila, silte e areia, respectivamente; densidade de partículas de $2,82 \mathrm{Mg} \mathrm{m}^{-3}$; densidade do solo de $1,39 \mathrm{Mg} \mathrm{m}^{-3} ; 0,15,0,26$ e $0,41 \mathrm{~m}^{3} \mathrm{~m}^{-3}$ de macro e microporosidade e porosidade total, respectivamente. O solo foi adubado para obtenção da produtividade esperada de soja, de 3,5 a 4,0 t ha $^{-1}$, segundo Raij et al. (1996).

O delineamento experimental foi inteiramente casualizado, com parcelas subdividas (seis níveis de compactação e quatro cultivares) e com quatro repetições, totalizando 96 subparcelas de $6,75 \mathrm{~m}^{2}$.

Antes da instalação do experimento foram realizadas uma escarificação do solo até $0,30 \mathrm{~m}$ de profundidade, uma gradagem niveladora, aplicação de calcário para elevar a saturação por bases para $60 \%$ e uma gradagem aradora até 0,12 m de profundidade. No início de novembro de 2003, quando o conteúdo de água estava próximo à capacidade de campo $(100 \mathrm{hPa})$, realizaram-se os tratamentos de compactação: $\mathrm{T}_{0}=0 ; \mathrm{T}_{1^{*}}=1 ; \mathrm{T}_{1}=1 ; \mathrm{T}_{2}=2 ; \mathrm{T}_{4}=4 ;$ e $\mathrm{T}_{6}=6$ passadas de um trator de $11 \mathrm{t}$ com os quatro pneus de mesma largura (0,40 m) e pressão interna, uma ao lado da outra, no sentido do declive da área, em toda a superfície do solo. No tratamento $\mathrm{T}_{1 *}$ a compactação ocorreu quando o solo estava 
mais seco para obter um nível menor de compactação. Por meio desses tratamentos, representou-se a condição de solo solto $\mathrm{T}_{0}$ até muito compactado $\mathrm{T}_{6}$.

Em dezembro, as sementes de soja (Glycine Max) das cultivares de ciclo precoce (120 dias): IAC Foscarin 31, e de ciclo médio (131-140 dias): MG/BR 46 (Conquista), BRS/ MG 68 (Vencedora) e IAC 8 - 2, foram inoculadas com Bradyrhizobium japonicum e semeadas na profundidade de 0,05 m, no espaçamento de 0,45 m entre linhas, no sentido transversal ao declive da área; após sete dias, realizou-se o desbaste mantendo-se 20 plantas por metro linear.

Após a semeadura fez-se o monitoramento do conteúdo de água no solo, até a colheita, em amostras coletadas nas camadas de 0,0 - 0,10 e 0,10 - 0,20 m (Gardner, 1986); foram, também, coletados dois grupos de seis amostras, com cilindros de $0,03 \mathrm{~m}$ de altura e $0,048 \mathrm{~m}$ de diâmetro $\left(53,16 \times 10^{-6} \mathrm{~m}^{3}\right)$, nas camadas de $0,03-0,06 ; 0,08-0,11$; 0,15 - 0,18; e $0,22-0,25 \mathrm{~m}$, para determinação dos atributos físicos, em cada tratamento; em seguida, uma amostra de cada grupo foram saturadas durante 24 horas e submetidas às tensões de 60; 100; 330; 600; 3000; e 15000 HPa em câmaras de pressão com placas porosas de Richards (Klute, 1986); ao se atingir o equilíbrio, foram pesadas e determinada a resistência do solo à penetração (RP) em duas repetições por amostra, na camada de 0,01 a $0,02 \mathrm{~m}$. A resistência à penetração foi determinada com o penetrômetro eletrônico estático, conforme descrito por Tormena et al. (1998) e Bengough et al. (2001); na seqüência, as amostras foram secadas para determinação do conteúdo de água em cada tensão (Gardner, 1986) e da densidade do solo (Blake \& Hartge, 1986).

Para definição do intervalo hídrico ótimo (IHO), a curva de retenção de água no solo foi ajustada através do modelo não linear, empregado por Silva et al. (1994), na forma logtransformada, descrita a seguir:

$$
\ln \theta=\mathrm{a}+\mathrm{bDs}+\mathrm{cln} \Psi
$$

A curva de resistência do solo à penetração foi ajustada utilizando-se o modelo não linear proposto por Busscher (1990), na forma log-transformada, a seguir:

$$
\ln R P=\operatorname{lnd}+\operatorname{eln} \theta+\text { flnDs }
$$

em que $\theta$ é o conteúdo volumétrico de água; Ds é a densidade do solo; $\Psi$ é a tensão de água no solo (hPa); RP é a resistência do solo à penetração; a, b, c, d, e e f, são os parâmetros dos ajustes dos modelos.

A partir dos valores estimados dos parâmetros, aplicouse o antilogaritmo e se obtiveram os valores estimados dos atributos físicos; desta maneira, assumindo o conteúdo de água na capacidade de campo equivalente à tensão de $100 \mathrm{hPa}$ e o ponto de murcha permanente de $15000 \mathrm{hPa}$, a $\theta_{\mathrm{CC}}$ e $\theta_{\mathrm{PMP}}$ foram estimadas pelas Eqs. 3 e 4, respectivamente, obtidas da Eq. 1 a seguir:

$$
\begin{gathered}
\theta_{\mathrm{CC}}=(\mathrm{a}+\mathrm{bDs}) * 100^{\mathrm{c}} \\
\theta_{\mathrm{PMP}}=(\mathrm{a}+\mathrm{bDs}) * 15000^{\mathrm{c}}
\end{gathered}
$$

O conteúdo de água a partir do qual a RP é limitante, foi obtido pela expressão 5, oriunda da Eq. 2:

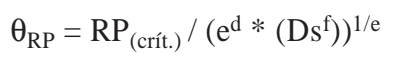

O conteúdo de água em que a porosidade de aeração $\theta_{\mathrm{PA}}$ é igual a 10\%, foi calculado pela Eq. 6 a seguir:

$$
\theta_{\mathrm{PA}}=[1-(\mathrm{Ds} / \mathrm{Dp})]-0,1
$$

A produtividade das cultivares de soja foi avaliada em área útil de $2,70 \mathrm{~m}^{2}$.

Os resultados foram submetidos a análise de variância, as médias comparadas pelo teste de Tukey a 5\% de probabilidade e realizadas análises de regressão entre a resistência do solo à penetração, determinada no conteúdo de água retida na capacidade de campo (100 hPa) e a produtividade de soja, utilizando-se o Statistical Analysis System (SAS, 1999) para realização das análises estatísticas.

\section{RESULTADOS E DISCUSSÃO}

Os valores dos atributos físicos do solo utilizados para o cálculo do intervalo hídrico ótimo (IHO) apresentaram ampla distribuição e os parâmetros dos modelos ajustados foram significativos a $1 \%$ de probabilidade (Tabela 1 ); esta situação indica confiabilidade dos dados para obtenção do IHO, mesmo partindo do princípio de que a amplitude e a precisão do IHO dependem também dos valores críticos de porosidade de aeração, conteúdo de água e resistência do solo à penetração (RP), adotados como impeditivos ao crescimento das plantas. Dentre esses atributos físicos, a RP é o que mais freqüentemente compromete o IHO, uma vez que aumenta com a compactação do solo (Silva et al., 1994; Tormena et al., 1998, 1999a, b; Beutler, 2003). O valor da RP adotado como limitante é de 2,0 MPa (Taylor et al., 1966; Tormena et al., 1999a, b), porém varia com o tipo de solo e cultura (Dexter, 1987; Beutler, 2003); assim, determinou-se este valor de RP limitante para a produtividade de cada uma das quatro cultivares de soja (Tabela 2) inserindo-o no IHO.

Em relação aos valores de RP limitantes, verificou-se que, individualmente, cada valor foi superior a 2,0 MPa (Tabela 2) e adotado como impeditivo ao crescimento radicular das plantas (Tormena et al., 1999a). O valor de RP a partir da qual a produtividade começou a decrescer, de 2,97, 2,95 e 2,71 MPa para as cultivares Vencedora, Conquista e IAC 8-2, respectivamente, foram superiores comparados com os da IAC Foscarim 31 (2,24 MPa). Esse fato ocorreu principalmente em decorrência de uma intensa infestação de ferrugem asiática (Phakopsora pachyrhizi), que causou maior queda de folhas nos tratamentos menos compactados $\left(\mathrm{T}_{0}\right.$ e $\left.\mathrm{T}_{1^{*}}\right)$, nas três primeiras cultivares, conforme indicado na Tabela 3; desta forma, a redução da produtividade em função da compactação do solo foi verificada apenas a partir do $T_{1}$, visto que essas três cultivares de ciclo médio estavam na fase de início de enchimento de grãos, severamente prejudicado pela queda de folhas e conseqüente redução da fotossíntese (Tabela 2). 
Tabela 1. Distribuição estatística dos atributos físicos utilizados nos ajustes dos modelos do intervalo hídrico ótimo, e coeficientes resultantes dos ajustes lineares da curva de retenção de água no solo $(\ln \theta=\ln a+b \ln D s+c \ln \Psi)$ e da curva de resistência do solo à penetração $(\operatorname{lnRP}=\ln d+\operatorname{eln} \theta+f \ln D s)$

\begin{tabular}{cccc}
\hline \multicolumn{1}{c}{ Atributo } & $\mathbf{N}$ & Mínimo & Máximo \\
Conteúdo de água, $\mathrm{m}^{3} \mathrm{~m}^{-3}(\theta)$ & 288 & 0,112 & 0,304 \\
Densidade do solo, $\mathrm{Mg} \mathrm{m}^{-3}(\mathrm{Ds})$ & 288 & 1,24 & 1,79 \\
Resistência à penetração, $\mathrm{MPa}(\mathrm{RP})$ & 288 & 0,80 & 20,13 \\
Tensão de água, hPa $(\Psi)$ & - & 60 & 15000 \\
\hline Parâmetro & Valor & Erro padrão & Prob. \\
\hline a & $-1,1461$ & 0,0375 & 0,0001 \\
\hline $\mathrm{b}$ & 0,7870 & 0,0686 & 0,0001 \\
$\mathrm{c}$ & $-0,1237$ & 0,0034 & 0,0001 \\
$\mathrm{~d}$ & $-5,6169$ & 0,2432 & 0,0001 \\
$\mathrm{e}$ & $-2,2841$ & 0,1037 & 0,0001 \\
$\mathrm{f}$ & 7,4046 & 0,3124 & 0,0001 \\
\hline
\end{tabular}

Tabela 2. Equações de regressão entre a resistência do solo à penetração (RP) e a produtividade das cultivares de soja e valor de RP, a partir do qual a produtividade decresce no Latossolo Vermelho

\begin{tabular}{|c|c|c|c|c|}
\hline Cultivar & $\begin{array}{c}\text { Equação } \\
y=\text { produtividade; } \\
x=R P\end{array}$ & $\mathrm{R}^{2}$ & $\begin{array}{l}\text { Produção } \\
\left(t \text { ha }^{-1}\right)^{(2)}\end{array}$ & $\begin{array}{c}\mathrm{RP}^{(3)} \\
(\mathrm{MPa})\end{array}$ \\
\hline IAC Foscarin31 & $y=1,4+0,8 x-0,2 x^{2}$ & $0,9 * *$ & 2,3 & $2,2^{(1)}$ \\
\hline MG/BR 46 Conquista & $y=-0,6+1,3 x-0,2 x^{2}$ & $0,7^{* *}$ & 1,3 & 2,9 \\
\hline BRS/MG68 Vencedora & $y=0,5+0,8 x-0,1 x^{2}$ & $0,8^{* *}$ & 1,7 & 2,9 \\
\hline IAC 8 - 2 & $y=0,01+0,9 x-0,2 x^{2}$ & $0,9 * *$ & 1,3 & 2,7 \\
\hline
\end{tabular}

** Significativo a $1 \%$ de probabilidade. ${ }^{1}$ Valor de resistência do solo à penetração, no conteúdo de água retida na capacidade campo $(100 \mathrm{hPa}) .{ }^{2}$ Produção máxima. ${ }^{3}$ Resistência à penetração a partir da qual a produção começou a decrescer

Valores superiores a 2,0 MPa de RP limitante à produtividade de soja, são ainda verificados, geralmente em estudos conduzidos em anos mais secos, visto que um pouco de compactação é benéfico por aumentar a área de contato das raízes com o solo, a condutividade hidráulica em função da umidade e a absorção de água e nutrientes pelas plantas (Barber, 1994). Este efeito também foi verificado por Ekwue \& Stone (1995), em milho. O decréscimo de produtividade de soja apenas a partir da RP de 2,24 a 2,97 MPa, também pode ter sido em função da estiagem que ocorreu no final do mês de dezembro de 2003 e na primeira quinzena dos meses de fevereiro e março de 2004 (Figura 1A e 1B). O conteúdo gravimétrico de água foi semelhante entre os tra-

Tabela 3. Média do nível de desfolha de quatro cultivares de soja (05/ 03/2004), transformados em $\sqrt{x+0,5}$, em níveis de compactação do Latossolo Vermelho, 15 dias após o aparecimento dos sintomas visíveis

\begin{tabular}{lcccccc}
\hline \multirow{2}{*}{ Cultivar } & \multicolumn{6}{c}{ Passadas do trator (11 t) } \\
\cline { 2 - 7 } & $\mathrm{T}_{0}$ & $\mathrm{~T}_{1^{*}}$ & $\mathrm{~T}_{1}$ & $\mathrm{~T}_{2}$ & $\mathrm{~T}_{4}$ & $\mathrm{~T}_{6}$ \\
IAC Foscarim 31 & $2,1 \mathrm{bc}{ }^{(1)}$ & $2,3 \mathrm{a}$ & $2,3 \mathrm{ab}$ & $2,1 \mathrm{bc}$ & $2,1 \mathrm{bc}$ & $2,0 \mathrm{c}$ \\
MG/BR46 Conquista & $2,3 \mathrm{a}$ & $2,2 \mathrm{a}$ & $1,9 \mathrm{~b}$ & $1,9 \mathrm{~b}$ & $1,9 \mathrm{~b}$ & $1,7 \mathrm{~b}$ \\
BRS/MG68 Vencedora & $2,2 \mathrm{a}$ & $2,1 \mathrm{ab}$ & $1,9 \mathrm{c}$ & $1,9 \mathrm{c}$ & $1,9 \mathrm{bc}$ & $1,9 \mathrm{c}$ \\
IAC 8 - 2 & $2,1 \mathrm{a}$ & $2,1 \mathrm{a}$ & $2,0 \mathrm{bc}$ & $2,0 \mathrm{ab}$ & $1,7 \mathrm{c}$ & $1,7 \mathrm{c}$ \\
\hline 1 Médias seguidas pela mesma letra, na linha, não diferem pelo teste de Tukey a 5\% de probabilidade. \\
$2 \mathrm{~T}_{0}=0 ; \mathrm{T}_{1 *}=1 ; \mathrm{T}_{1}=1 ; \mathrm{T}_{2}=2 ; \mathrm{T}_{4}=4$ e $\mathrm{T}_{6}=6$ passadas de um trator de 11 t, no mesmo local, no \\
conteúdo de água próximo à capacidade de campo. No tratamento $\mathrm{T}_{1^{*} \text { o tráfego foi realizado quando 0 }}$ \\
solo estava mais seco.
\end{tabular}

tamentos (Figura 1C); entretanto, no final do mês de dezembro de 2003, o conteúdo de água foi inferior ao ponto de murcha permanente - PMP (Figura 1B), provocando à senescência de plantas.

Por outro lado verifica-se, com base na Tabela 4, que a produtividade de soja nos $\mathrm{T}_{0}$ e $\mathrm{T}_{1^{*}}$ foi igual à do tratamento mais compactado $\mathrm{T}_{6}$, em que a RP foi de 4,42 MPa, exceto na cultivar IAC Foscarim 31. Ao admitir que o valor de RP de 4,42 MPa é restritivo ao desenvolvimento das plantas (Taylor et al., 1966; Barber, 1994; Beutler, 2003), evidencia-se que a ferrugem asiática causou maior decréscimo da produtividade de soja nos tratamentos com menores níveis de compactação do solo.

Desta forma, não foi possível estabelecer um ótimo ajuste das equações quadráticas de regressão, fato que dificultou a determinação exata do valor de RP e Ds, a partir do qual a produtividade de soja decresceu; inclusive, as equações de regressão da densidade do solo com a produtividade de soja não foram significativas sendo, assim, apresentado o teste de Tukey para comparar as médias.

Ao se plotar no IHO os valores de RP limitantes para cada cultivar (Tabela 2), obtiveram-se a amplitude do IHO e a densidade do solo crítica (Dsc) (Figura 2) para todas as cultivares. A amplitude do IHO variou com o valor de RP limitante inserido no modelo, decrescendo a partir da Ds de 1,22 (IAC Foscarim 31) a 1,28 $\mathrm{Mg} \mathrm{m}^{-3}$ (Vencedora e IAC 8-2), corroborando com Tormena et al. (1999b) e Beutler et al. (2004).

A partir desses valores e segundo Silva \& Kay (1997), aumenta a possibilidade de ocorrência de conteúdo de água no solo restritivo ao crescimento radicular, até atingir a Dsc, quando então as condições são altamente restritivas ao crescimento radicular.

Segundo os modelos do IHO (Figura 2), a $\theta_{\mathrm{PA}}$ foi superior a $\theta_{\mathrm{CC}}$ e não limitante ao crescimento radicular, corroborando com Tormena et al. (1999b) e Leão et al. (2004); também, não foi limitante devido, sem dúvida, ao fato de que a capacidade de campo é atingida normalmente dois dias após a precipitação pluvial (Reicherdt, 1985) a qual foi, em geral, intensa e esparsa, durante o ciclo da cultura (Figura 1). Por sua vez, as plantas foram submetidas a longos períodos com menor conteúdo de água no solo e, assim, o impedimento mecânico do solo ao crescimento radicular foi o fator restritivo ao seu desenvolvimento. A RP $\left(\theta_{\mathrm{RP}}\right)$ foi o atributo físico que causou decréscimo drástico na amplitude do IHO a partir da Ds de 1,22 (IAC Foscarim 31) a 1,28 $\mathrm{Mg} \mathrm{m}^{-3}$ (Vencedora e IAC 8-2), condição de solo descompactado (solto), até a densidade do solo crítica ao desenvolvimento radicular (Dsc) (Figura 2), corroborando com Tormena et al. (1999b), Beutler (2003), Beutler et al. (2004) e Leão et al. (2004), em solos tropicais. Este decréscimo da amplitude do IHO a partir da Ds de 1,22 $\mathrm{Mg} \mathrm{m}^{-3}$, ocorre em função da relação direta da RP com a Ds e inversa com a $\theta$ (Hamblim, 1985); deste modo, o aumento da Ds causa maior RP e, quando o valor da RP é o valor limite à produtividade, o decréscimo no conteúdo de água aumenta a RP e expõem a planta a condições adversas ao crescimento, i.e. menor conteúdo de água no solo que a retida na tensão de 100 hPa na Dsc. 

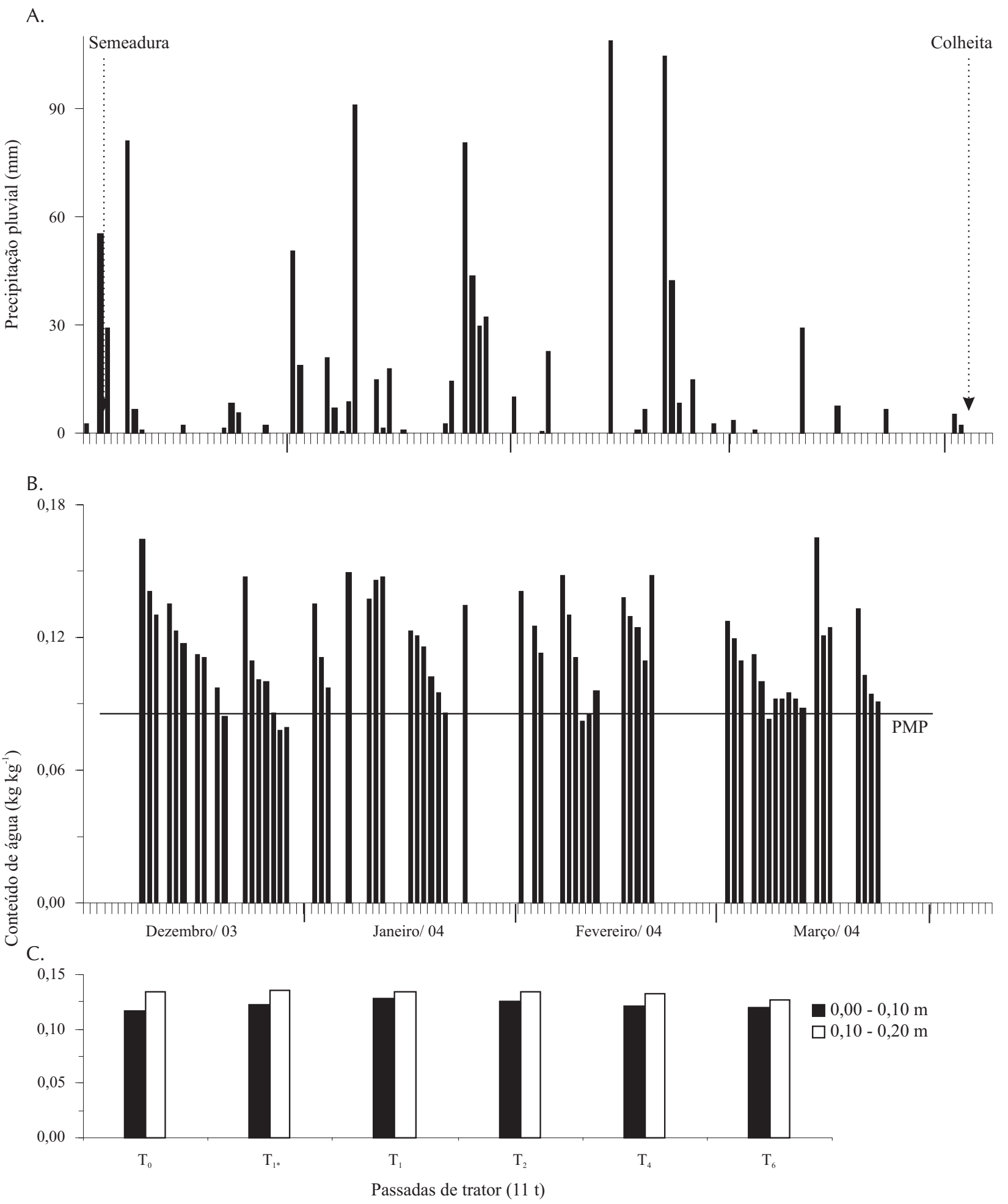

Figura 1. Precipitação pluvial diária (A), conteúdo gravimétrico de água na camada de $0,0-0,2 \mathrm{~m}$ durante o cultivo da soja (B) e conteúdo gravimétrico de água nos diferentes tratamentos (média de quatro dias) (C), no Latossolo Vermelho

Tabela 4. Produtividade média das cultivares de soja (t ha-1) em função da densidade do Latossolo Vermelho

\begin{tabular}{lllllll}
\hline \multirow{2}{*}{ Cultivar } & \multicolumn{5}{c}{ Densidade do Solo $\mathbf{~ ( M g ~ m ~}^{-3}$ ) } \\
\cline { 2 - 7 } IAC Foscarim 31 & $\mathbf{1 , 3 9}$ & $\mathbf{1 , 5 6}$ & $\mathbf{1 , 6 7}$ & $\mathbf{1 , 6 9}$ & $\mathbf{1 , 6 8}$ & $\mathbf{1 , 7 0}$ \\
MG/BR 46 (Conquista) & $2,065 \mathrm{ab}$ & $2,265 \mathrm{ab}$ & $2,470 \mathrm{a}$ & $1,969 \mathrm{bc}$ & $1,859 \mathrm{bc}$ & $1,543 \mathrm{c}$ \\
BRS/MG 68 (Vencedora) & $0,668 \mathrm{c}$ & $0,823 \mathrm{bc}$ & $1,601 \mathrm{a}$ & $1,165 \mathrm{~b}$ & $1,075 \mathrm{bc}$ & $0,929 \mathrm{bc}$ \\
IAC 8 - 2 & $1,299 \mathrm{~b}$ & $1,392 \mathrm{ab}$ & $1,819 \mathrm{a}$ & $1,606 \mathrm{ab}$ & $1,595 \mathrm{ab}$ & $1,446 \mathrm{ab}$ \\
\hline
\end{tabular}

Médias seguidas pela mesma letra minúscula na linha não diferem entre si pelo teste de Tukey a $5 \%$ de probabilidade 

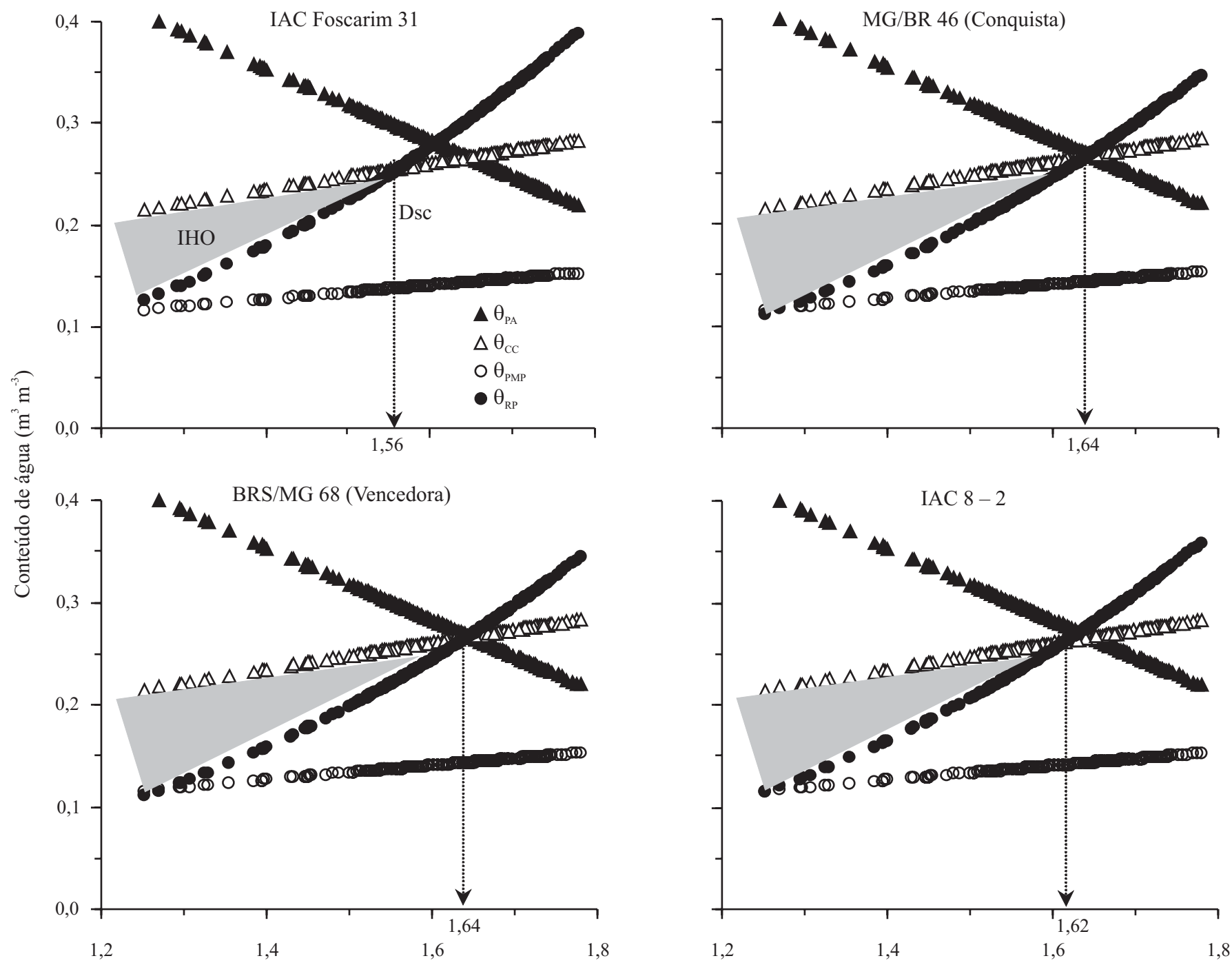

Densidade do solo $\left(\mathrm{Mg} \mathrm{m}^{-3}\right)$

Figura 2. Intervalo hídrico ótimo para as cultivares de soja, em Latossolo Vermelho

A Dsc variou de 1,56 (IAC Foscarin 31) a 1,64 $\mathrm{Mg} \mathrm{m}^{-3}$ (Vencedora e IAC 8 - 2). A partir desses valores de Ds e, segundo o modelo do IHO, a qualidade física do solo ao crescimento radicular é crítica, mesmo com disponibilidade de água (Weaich et al., 1992), já que o IHO ao crescimento radicular é igual a zero (Silva et al., 1994); então, a partir desta Ds, são imprescindíveis as adoções de medidas biológicas ou mecânicas de descompactação do solo, para evitar decréscimos de produtividade.

Utilizando-se os valores limitantes dos atributos físicos diretamente relacionados ao crescimento das plantas no modelo do IHO, supõem-se que os valores de Dsc serão semelhantes aos valores de Ds, a partir dos quais a produtividade do campo decresce, verificados na Tabela 3; porém os valores de Ds limitantes à produtividade de soja no campo foram superiores a Dsc obtida no IHO e variaram de 1,67 a $1,70 \mathrm{Mg} \mathrm{m}^{-3}$, evento este relacionado, possivelmente, ao fato do intenso ataque da ferrugem asiática, conforme já referido, o que impossibilitou a obtenção exata dos valores de Ds restritivos à produtividade de soja. Para o solo estudado com teor de argila de $330 \mathrm{~g} \mathrm{~kg}^{-1}$, por meio do diagrama apresentado em Dexter (2004), o valor de Ds limitante ao crescimento radicular das culturas é de 1,62 $\mathrm{Mg} \mathrm{m}^{-3}$; Beutler (2003) verificou, para o mesmo solo, que a produtividade de soja decresceu a partir da Ds de $1,48 \mathrm{Mg} \mathrm{m}^{-3}$, no ano anterior, com melhor distribuição da precipitação pluvial durante o ciclo da cultura.

\section{CONCLUSÕES}

1. A densidade do solo crítica à produtividade de soja, obtida no modelo do IHO, varia entre as cultivares de 1,56 a 1,64 $\mathrm{Mg} \mathrm{m}^{-3}$, em Latossolo Vermelho.

2. Os valores de densidade do solo crítica determinados no modelo do IHO foram inferiores aos da densidade do solo, a partir das quais a produtividade das cultivares de soja decresceu. 


\section{AGRADECIMENTOS}

O primeiro autor agradece à FAPESP pela bolsa de PósDoutorado, e o segundo e o terceiro ao CNPq, pela bolsa de pesquisador.

\section{LITERATURA CITADA}

Barber, R. G. Persistence of loosened horizons and soybean yield increases in Bolivia. Soil Science Society of America Journal, Madison, v.58, n.3, p.943-950, 1994.

Bengough, A. G.; Campbell, D. J.; O’sullivan, M. F. Penetrometer techniques in relation to soil compaction and root growth. In: Soil environmental analysis: Physical Methods. 2.ed. Nova York: Marcel Dekkee, 2001. p.377-403.

Beutler, A. N. Efeito da compactação do solo e do conteúdo de água nas características agronômicas de soja e arroz de sequeiro. Jaboticabal: UNESP, 2003. 126p. Tese Doutorado

Beutler, A. N.; Centurion, J. F.; Silva, A. P. Intervalo hídrico ótimo e a produção de soja e arroz em dois latossolos. Irriga, Botucatu, v.9, n.2, p.181-192, 2004.

Blake, G. R.; Hartge, K. H. Bulk density. In: Klute, A. (ed.). Methods of soil analysis: physical and mineralogical methods. 2.ed. Madison: American Society of Agronomy, 1986. v.1. p.363-375.

Boone, F. R. Toward soil compaction limits for crop growth. Netherlands Journal of Agricultural Science, The Hague, v.34, n.3, p.349-360, 1986.

Busscher, W. J. Adjustment of flat-tipped penetrometer resistance data to a common water content. Transactions of the ASAE, Saint Joseph, v.33, n.2, p.519-524, 1990.

Dexter, A. R. Mechanics of root growth. Plant and Soil, The Netherlands, v.98, n.3, p.303-312, 1987.

Dexter, A. R. Soil physical quality: Part I. Theory, effects of soil texture, density, and organic matter, and effects on root growth. Geoderma, Amsterdam, v.120, n.3, p.201-214, 2004.

Ekwue, E. I.; Stone, R. J. Irrigation scheduling for sweet maize relative to soil compaction conditions. Journal of Agricultural Engineering Research, London, v.62, n.2, p.85-94, 1995.

Gardner, W. E. Water content. In: Klute, A. (ed.). Methods of soil analysis: Physical and mineralogical methods. 2.ed. Madison: American Society of Agronomy, 1986. p.493-544.

Gee, G. W.; Bauder, J. W. Particle-size analysis. In: Klute, A. (ed.). Methods of soil analysis. 2.ed. Madison: American Society of Agronomy, Part. 1. 1986. p.383-411.

Grable, A. R.; Siemer, E. G. Effects of bulk density, aggregate size, and soil water suction on oxygen diffusion, redox potential and elongation of corns roots. Soil Science Society of America Journal, Madison, v.32, p.180-186, 1968.

Hamblim, A. P. The influence of soil structure on water movement, crop root growth and water uptake. Advances in Agronomy, San Diego, v.38, n.1, p.95-158, 1985.

Klute, A. Water retention: laboratory methods. In: Klute, A. (ed.). Methods of soil analysis. 2.ed. Madison: American Society of Agronomy, 1986. Part. 1, p. 635-662.
Leão, T. P.; Silva, A. P.; Macedo, M. C. M.; Imhoff, S.; Euclides, V. P. B. Intervalo hídrico ótimo na avaliação de sistemas de pastejo contínuo e rotacionado. Revista Brasileira de Ciência do Solo, Viçosa, v.28, n.3, p.415-423, 2004.

Letey, J. Relationship between soil physical properties and crop production. Advances in Soil Science, New York, v.1, n.1, p.277-294, 1985.

Nelson, W. E.; Rahi, G. S.; Reeves, L. Z. Yield potential of soybean as related to soil compaction induced by farm traffic. Agronomy Journal, Madison, v.67, n.6, p.769-772, 1975.

Raij, B. van; Cantarella, H.; Quaggio, J. H.; Furlani, A. M. C. Recomendação de adubação e calagem para o estado de São Paulo. 2.ed. Campinas: Instituto Agronômico e Fundação IAC, 1996. 285p. Boletim Técnico, 100

Reichardt, K. Processos de transferência no sistema solo planta atmosfera. Piracicaba: Fundação Cargill, 1985. 445p.

Reichardt, K. Capacidade de campo. Revista Brasileira de Ciência do Solo, Campinas, v.12, n.3, p.211-216, 1988.

Savage, M. J.; Ritchie, J. T.; Land, W. L.; Dugas, W. A. Lower limit of soil water available. Agronomy Journal, Madison, v.88, n.5, p.844-851, 1996.

Silva, A. P.; Kay, B. D. The sensitivity of shoot growth of corn to the least limiting water range of soils. Plant and Soil, Dordrecht, v.184, n.2, p.323-329, 1996.

Silva, A. P.; Kay, B. D. Effect of soil water content variation on the least limiting water range. Soil Science Society of America Journal, Madison, v.61, n.3, p.884-888, 1997.

Silva, A. P.; Kay, B. D.; Perfect, E. Characterization of the least limiting water range. Soil Science Society of America Journal, Madison, v.58, n.6, p.1775-1781, 1994.

SAS - Statistical Analysis System Institute. SAS/STAT Procedure Guide for Personal Computers. 5. ed. Cary, NC:SAS Inst. 1999. 334p.

Taylor, H. M.; Roberson, G. M.; Parker Jr., J. J. Soil strength-root penetration relations to medium to coarse-textured soil materials. Soil Science, Baltimore, v.102, n.1, p.18-22, 1966.

Tormena, C. A.; Silva, A. P.; Gonçalves, A. C. A.; Folegatti, M. V. Intervalo ótimo de potencial da água no solo: Um conceito para avaliação da qualidade física do solo e manejo da água na agricultura irrigada. Revista Brasileira de Engenharia Agrícola Ambiental, Campina Grande, v.3, n.3, p.286-292, 1999a.

Tormena, C. A.; Silva, A. P.; Libardi, P. Caracterização do intervalo hídrico ótimo de um Latossolo Roxo sob plantio direto. Revista Brasileira de Ciência do Solo, Viçosa, v.22, n.4, p.573581, 1998.

Tormena, C. A.; Silva, A. P.; Libardi, P. L. Soil physical quality of a Brazilian Oxisol under two tillage systems using the least limiting water range approach. Soil \& Tillage Research, Amsterdam, v.52, n.3, p.223-232, 1999 b.

Weaich, K.; Bristow, K. L.; Cass, A. Pre-emergent shoot growth of maize under different dryin conditions. Soil Science Society of America Journal, Madison, v.56, n.4, p.1272-1278, 1992.

Zou, C.; Sands, R.; Buchan, G.; Hudson, I. Least limiting water range: a potential indicator of physical quality of forest soils. Australian Journal of Soil Research, Sydney, v.38, n.5, p.947958, 2000. 